\title{
Markus Friedrich, Die Jesuiten. Aufstieg, Niedergang, Neubeginn
}

\section{David Aeby}

\section{OpenEdition}

\section{Journals}

Édition électronique

URL : http://journals.openedition.org/ifha/9045

DOI : $10.4000 /$ ifha. 9045

ISSN : 2198-8943

\section{Éditeur}

IFRA - Institut franco-allemand (sciences historiques et sociales)

\section{Référence électronique}

David Aeby, " Markus Friedrich, Die Jesuiten. Aufstieg, Niedergang, Neubeginn », Revue de l'IFHA [En ligne], Date de recension, mis en ligne le 07 juin 2018, consulté le 24 septembre 2020. URL : http:// journals.openedition.org/ifha/9045; DOI : https://doi.org/10.4000/ifha.9045

Ce document a été généré automatiquement le 24 septembre 2020

(CIFHA 


\title{
Markus Friedrich, Die Jesuiten. Aufstieg, Niedergang, Neubeginn
}

\author{
David Aeby
}

\section{RÉFÉRENCE}

Markus Friedrich, Die Jesuiten. Aufstieg, Niedergang, Neubeginn, München/Berlin/Zürich: Piper, 2016, 727 p., $39 €$ 
L'historiographie de la Compagnie de Jésus a connu depuis les années 1990 un renouveau qui a fait de l'histoire des jésuites une voie d'accès particulièrement fructueuse pour l'intelligence du monde moderne. En 2011, Markus Friedrich avait déjà publié une étude précisément documentée sur l'organisation administrative de l'ordre jésuite, de sa fondation jusqu'à sa suppression en 1773 (Der lange Arm Roms? Globale Verwaltung und Kommunikation im Jesuitenorden 1540-1773, 2011). Dans ce nouveau livre, l'historien entreprend une histoire globale de la Compagnie. L'essentiel de l'ouvrage traite de l'«ancienne Compagnie ", soit de la période précédent la suppression. Toutefois, une partie du dernier chapitre est consacrée aux activités des jésuites entre 1773 et la

M A R K U S F R I E D R I C H
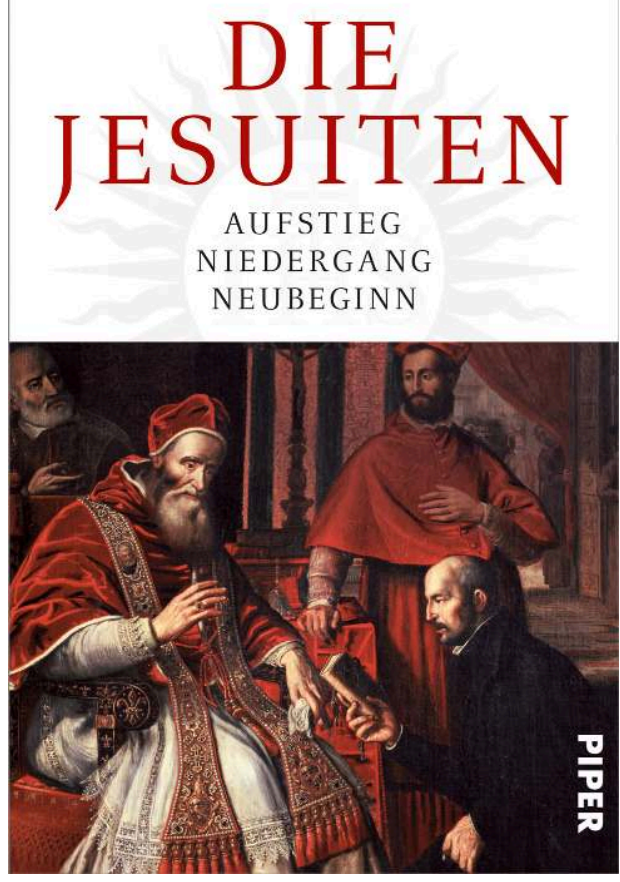
restauration de la Compagnie en 1814.

Enfin, un épilogue expose les grandes évolutions de l'ordre au cours des XIX et XX siècles, jusqu'à l'élection au pontificat du jésuite Bergoglio.

M. Friedrich ne cache pas la difficulté de son projet : écrire l'histoire des jésuites, c'est écrire en miniature une histoire du monde (p. 22). L'auteur a donc construit son étude autour des thèmes qu'il a jugés centraux dans l'histoire de la Compagnie, tenant compte de la périodisation inhérente à chacun d'eux et s'autorisant parfois des arrêts sur des sujets dont l'importance plus secondaire pouvait en éclairer de plus déterminants.

La présentation des structures de la Compagnie et de leur mise en place pointe les difficultés dues à la croissance fulgurante de l'institution. L'écart entre les exigences des supérieurs garants de l'idéal des textes fondateurs et les attentes des religieux entrés dans l'ordre pour des raisons variées a conduit à des désillusions et à des déceptions de part et d'autre, que le discours hagiographique laisse en principe de côté. Ainsi, les supérieurs romains ont fréquemment dû lutter contre des sentiments nationaux contraires au désir d'uniformité des premiers jésuites (p. 172-173).

Dans les chapitres suivants, l'auteur s'intéresse à l'inscription de la Compagnie dans le monde moderne. Dans une chrétienté, et plus particulièrement une catholicité, où s'affrontaient différentes visions de l'orthodoxie, l'ordre a dû trouver une place parmi des traditions déjà anciennes au moment de sa fondation, et les tensions avec d'autres ordres religieux ont été fréquentes. Ces tensions ont également traversé la Compagnie elle-même. Alors que la casuistique représentait, pour un ordre fondamentalement apostolique destiné au "salut des âmes", une activité de première importance, les casuistes jésuites, ont été partagés entre plusieurs courants (p. 171-172). Dépassant de loin les cercles du monde religieux, les jésuites se sont saisis d'un monde dont les sphères obéissaient à des logiques parfois contradictoires. M. Friedrich montre comment la Compagnie a cherché à construire et à préserver son identité, tout en se 
confrontant et en participant à la vie de ces sphères politiques, économiques et scientifiques. Des cas individuels, tels celui de Ferdinand Orban, collectionneur au service du prince-électeur palatin (p.315), illustrent les limites de la curie généralice face à des forces centrifuges soutenues de l'extérieur.

Enfin, l'auteur s'intéresse à la projection géographiquement et culturellement la plus lointaine de l'ordre : les missions étrangères. Là encore, M. Friedrich corrige une image trop lisse de la Compagnie et souligne l'inégale présence jésuite à travers le monde colonial et surtout une répartition des ressources humaines très disproportionnée entre les religieux voués à l'apostolat auprès des colons et la minorité des missionnaires envoyés chez les indigènes. Le dernier chapitre du livre revient sur la chute de la Compagnie en 1773, dont l'auteur replace la dynamique événementielle dans la perspective du temps plus long du discours antijésuite. Il souligne également la passivité des religieux durant les années précédant le bref Dominus ac Redemptor, dont il propose d'imputer une part de responsabilité au conservatisme du préposé général Ricci (p. 346-347). La restauration de l'ordre est replacée dans le contexte postrévolutionnaire et les dynamiques des différents groupes décidés à faire renaitre la Compagnie. Là encore, les avis divergeaient sur l'orientation à donner à une institution que l'auteur juge être réapparue en 1814 sans avoir subie de véritables réformes (p. 566). Ces dernières surviendront plus tard, les plus profondes au cours du second $\mathrm{XX}^{\mathrm{e}}$ siècle.

Si l'appareil critique comporte de fréquents renvois à des sources de premières mains, l'essentiel de cette synthèse - comme attendu pour un tel travail - repose sur la littérature secondaire, que l'auteur a le grand mérite d'avoir travaillée dans des historiographies de langues et d'écoles très variées. Malgré son classement alphabétique, l'imposante bibliographie à la fin de l'ouvrage sera utile pour les futures études jésuites.

Dans son introduction, M. Friedrich annonce son intention de montrer la diversité de la Compagnie (p. 23). C'est ce qu'il parvient à faire de manière absolument convaincante au fil de ce livre. En mettant en regard les textes normatifs de l'ordre mais, aussi le discours de ses adversaires, les grandes orientations de la Compagnie, mais aussi des parcours individuels, il nous présente la richesse d'une histoire trop souvent réduite à quelques clichés. Il devient dès lors difficile de parler de la Compagnie de Jésus comme d'un bloc monolithique. Plus qu'une synthèse, Die Jesuiten invite donc à rouvrir des dossiers particuliers et des études de cas liés à l'histoire des jésuites et à les examiner dans leurs particularités.

\section{INDEX}

Index chronologique : Période moderne

Thèmes : Histoire religieuse 
AUTEURS

DAVID AEBY

EHESS /Université Fribourg 